\title{
Analysis of TCM Syndrome Types of Manic Disorder: Data from Clinical Therapy of TCM and Western Medicine
}

\author{
Sun Fengli ${ }^{1}$, Li Wei ${ }^{1}$, Yu Fang ${ }^{1}$, Song Xinyu ${ }^{1}$, Liu Jie $^{2}$, Chen Fengpei ${ }^{2}$, Zhu Jianfeng ${ }^{2}$, Gao Zhihan ${ }^{3}$, \\ Ma Yongchun ${ }^{1}$, Chen Jiong ${ }^{1}$, Xing Baoping ${ }^{1}$, Chen Song ${ }^{4}$, Jin Weidong ${ }^{1,5}$, , Wang Zhiqiang ${ }^{6, *}$ \\ ${ }^{1}$ Department of Psychiatry, Zhejiang Province Mental Health Center, Zhejiang Province Tongde Hospital, Hangzhou, China \\ ${ }^{2}$ The Second College, Zhejiang Chinese Medicine University, Hangzhou, China \\ ${ }^{3}$ Department of Clinical Psychology, Hangzhou Geriatric Hospital, Hangzhou, China \\ ${ }^{4}$ Mental Health Center, Medical College of Zhejiang University, Hangzhou, China \\ ${ }^{5}$ Zhejiang Chinese Medicine University, Hangzhou, China \\ ${ }^{6}$ Department of Psychosomatic, Chuiyangliu Hospital, Tsinghua University, Beijing, China \\ Email address: \\ shuimu1980314@sina.com (Sun Fengli),wdjin@163.com (Jin Weidong),wzq1965@126@.com (Wang Zhiqiang) \\ ${ }^{*}$ Corresponding author
}

\section{To cite this article:}

Sun Fengli, Li Wei, Yu Fang, Song Xinyu, Liu Jie, Chen Fengpei, Zhu Jianfeng, Gao Zhihan, Ma Yongchun, Chen Jiong, Xing Baoping, Chen Song, Jin Weidong, Wang Zhiqiang. Analysis of TCM Syndrome Types of Manic Disorder: Data from Clinical Therapy of TCM and Western Medicine. International Journal of Chinese Medicine. Vol. 4, No. 1, 2020, pp. 12-15. doi: 10.11648/j.ijcm.20200401.12

Received: December 22, 2019; Accepted: December 30, 2019; Published: February 14, 2020

\begin{abstract}
Objective: to analyze the distribution characteristics and composition ratio of Chinese medicine syndrome in manic disease from the published literature on the combined treatment of manic mania by Chinese and Western medicine. Methods: to collect literature from Chinese database, to analyze and classify the cases that meet the standard combined treatment of Chinese and Western medicine, analyze the distribution of different syndromes and composition ratio. Results: 1385 cases were studied. Among them, $48.95 \%$ were phlegm fire disturbance to the mind, 20.50\% were stagnation of phlegm and heat, $12.37 \%$ were liver and gall depression heat, $10.76 \%$ were qi stagnation and blood stasis, and $7.51 \%$ were fire injury Yin. Conclusion: The first three syndrome can cover more than $80 \%$ of the proportion. The contribution degree of the first three is also higher.
\end{abstract}

Keywords: Mania, Chinese Medicine Mania, Chinese Medicine Syndromes, Proportion, Contribution Degree

\section{Introduction}

Bipolar disorder $(\mathrm{BD})$ has a substantial lifetime prevalence in the population at $4 \%$. Because the manic or depressive symptoms of $\mathrm{BD}$ tend to be severe and recurrent over a patient's lifetime, the condition is associated with significant burden to the individual, caregivers, and society. Clinician awareness that BD may be present increases the likelihood of successful recognition and appropriate treatment. A number of pharmacological and nonpharmacological treatments are available for acute and maintenance treatments, with the prospect of achieving reduced symptom burden and increased functioning for many patients [1]. In bipolar disorder, there are two states of depression and mania, and they alternate or change each other. Mania is characterized by excitement, talkative, restless, emotional upsurge, impulse, energy and so on. Those who are serious may have violent tendency or behavior [2], which are all typical mania in traditional Chinese medicine (TCM), and so called psychotic mania or "TCM crazy" (Chinese language: Kuangbing) [3]. In many current clinical models about the treatment of mania with the combination of Chinese and Western medicine, almost all of them are diagnosed by western medicine first and then treated by syndrome differentiation of traditional Chinese medicine [4-6]. Among them, the mania related to this mania should be divided into several types which are suitable for clinical use [7, 8]. Although there is a saying that "all manias belong to fire", there are still some differences in the classification of mania syndrome types in TCM, no matter the number of syndrome types or the characteristics of syndrome types $[9,10]$. Therefore, we collected the literature of mania treated by combination of Chinese and Western medicine, analyzed and 
evaluated the TCM syndrome types of mania included in the literature, in order to evaluate the distribution characteristics and composition ratio of TCM syndrome types of mania.

\section{Materials and Methods}

\subsection{Inclusion Criteria}

(1) manic phase of bipolar disorder or manic phase diagnosed by the diagnostic criteria of manic or manic episode diagnosis in CCMD-3, ICD-10 or DSM-5; (2) The treatment mode is combination of TCM and Western medicine, (3) The combination of TCM and Western medicine must be study group and control group must be treated only by Western medicine; (4) The corresponding syndrome types of traditional Chinese medicine are only a TCM a syndrome type (5) The formula is unified with the syndrome types, and there can be addition and subtraction; (6) The observation time is not less than 4 weeks; (7) There are specific methods to evaluate the efficacy or severity by manic scale; (8) Our study just is the study group that be given the combination of traditional Chinese and Western medicine. The literature is only limited to the Chinese literature published in China Contributions, excluding Hong Kong, Macao and Taiwan literature.

\subsection{Literature Collection Method}

Searches were applied to the following electronic databases, but only in china: Chinese Biomedical Database (CBM), China National Knowledge Infrastructure (CNKI), WANFANG and Chinese Social Sciences Citation Index (VIP) databases. The search strategy was based on combination treatment of TCM and western medicine for mania The Search terms of literature retrieval are combination of Chinese and Western medicine, mania (bipolar disorder or affective psychosis). Then artificial screening was carried out, and the key words were mania (disease), traditional Chinese medicine.

\subsection{General Information}

There are a total of 33 research papers. Among them, 27 were single syndrome type and 6 were multi syndrome type $[11,12]$.

\subsection{Statistical Methods}

The number of cases and the proportion of each syndrome type in this study were calculated. Then we combine the similar syndrome types and count the average ratio of the same syndrome type and the overall composition ratio and contribution degree of each syndrome type.

\section{Results}

\subsection{The Information of all TCM Syndromes}

The 33 research papers were collected from Chinese dabase, which met our criteria. The TCM syndromes involved are phlegm fire disturbance to internal (Chinese language: Tanhuoneirao), phlegm fire disturbance to the heart (Chinese language: Tanhuoraoxin), phlegm fire disturbance to the mind (Chinese language: Tanhuoraoshen), liver and gall heat depression (Chinese language: gandanyure), qi stagnation and blood stasis (Chinese language: qizhixueyu), fire injury Yin (Chinese language: huoshengshangyin), yin deficiency and fire flourishing (Chinese language: yinxuhuowang), qi stagnation and fire melting (Chinese language: qiyuhuahuo), phlegm fire Yongsheng (Chinese language: tanhuoyongsheng), liver fire hyperactivity (Chinese language: ganhuokangsheng), phlegm heat (fire) stagnation (Chinese language: tanqiyujie/tanhuoyujie), Qi and blood stagnation (Chinese language: qixueningzhi) and phlegm qi stagnation (Chinese language: tangqiyujie). Among them, qi stagnation and fire melting was 67 cases $(1$, represent the number of studies, the same below), 149 cases (2) of phlegm fire Yongsheng, 268 cases (10) of phlegm fire internal disturbance, 18 cases (2) of yin deficiency and fire flourishing, 73 cases (3) of liver and gall heat depression, 86 cases (4) of fire injury Yin, 190 cases (4) of phlegm fire disturbance to the mind, 30 cases (1) of liver fire hyperactivity, 101 cases (3) of phlegm heat (fire) stagnation, 220 cases (5) of phlegm fire disturbance heart, 78 cases (2) of qi stagnation and blood stasis, 71 cases of Qi and blood stagnation (2), 34 cases of phlegm heat (fire) stagnation (1).

\subsection{The Similar Syndromes Were Merged}

The phlegm fire disturbance to the heart, phlegm fire disturbance to internal, phlegm fire disturbance to the mind are called by a joint name as phlegm fire disturbance to the mind (Chinese language: Tanhuoraoshen). Phlegm fire Yongsheng, phlegm heat depression, phlegm qi depression are similar and called by a joint name as phlegm heat stagnation (Chinese language: tanreyujie). Qi and blood stagnation is similar to stagnation of Qi and blood stasis and be called name as stagnation of Qi and blood stasis (Chinese language: qizhixueyu). Yin deficiency and fire flourishing are similar to fire injury Yin and are called by a joint name as fire injury Yin (Chinese language: huoshengshangyin), which are collectively referred to as fire hyperactivity and Yin injury. Qi stagnation and fire melting, liver fire hyperactivity, and liver and gall depression heat are all related to liver, which are collectively called a name of liver and gall depression heat (Chinese language: gandanyure). So there were 284 cases (6) of phlegm heat depression, 149 cases (4) of qi stagnation and blood stasis, 678 cases (19) of phlegm fire disturbance to the mind, 170 cases (5) of liver and gall depression heat, and 104 cases (6) of fire injury Yin. There were 1385 cases in total. Among them, 48.95\% were caused by phlegm fire disturbance to the mind, $20.50 \%$ by phlegm and heat stagnation, $12.37 \%$ were liver and gall depression heat, $10.76 \%$ were qi stagnation and blood stasis, and $7.51 \%$ were fire injury Yin. The first three syndrome accounted for $81.73 \%$ and the first four $92.49 \%$. 


\subsection{The Proportion and Contribution of Common TCM Syndromes}

There are 40 studies of all TCM syndromes in total. The proportion of the number of studies involved in the five syndromes was normalized Among them, $19 / 40=47.5 \%$ for phlegm fire disturbance to the mind, $6 / 40=15 \%$ for phlegm and heat stagnation, $6 / 40=15 \%$ for fire injury Yin, $5 / 40=$ $12.5 \%$ for liver and gall depression heat, and $4 / 40=10 \%$ for qi stagnation and blood stasis. The number of studies indicates the contribution degree that refer to Multiplying the proportion of styuties by the proportion of syndrome types. The contribution degree of phlegm fire disturbance to the mind is $0.4895 \times 0.475=0.233$, the contribution of phlegm heat stagnation is $0.205 \times 0.15=0.031$, the contribution of liver and gall depression heat is $0.1237 \times 0.125=0.015$, the contribution of fire injury Yin is $0.0751 \times 0.15=0.011$, the contribution of qi stagnation and blood stasis is $0.1076 \times 0.1=0.011$. The order of contribution degree was phlegm fire disturbance to the mind, phlegm and heat stagnation, liver and gall depression heat, qi stagnation and blood stasis, fire injury Yin.

\section{Discussion}

Traditional Chinese medicine pays special attention to the disturbance of phlegm and fire to the spirit in the understanding of mania or manic episode. The manic manifestations of bipolar disorder, such as exaggeration, talkative, sleep need reduction, mental wandering, thought running, behavior rashness, psychomotor excitement, control power reduction, disordered consumption, sexual behavior rashness and so on $[1,13]$, are very similar to those caused by phlegm fire disturbance to the mind in traditional Chinese medicine $[14,15]$, indicating that phlegm fire disturbance to the mind in manic syndrome in TCM are common. The results of this literature analysis also have similar conclusions. It has the most number of research projects and the largest proportion of composition, and the highest contribution degree.

Bipolar disorder is characterized by manic and depressive symptoms alternating at the same time or in different stages in the same patient. The excitement, impulse, agitation, aggression, irritability, racing thinks are common symptoms in manic stage and lower energy, less interest, slower thinks and suicide behavior is more in depressive stage $[1,11,13]$. There are some patients with mixed of depressive and manic symptoms [14, 15] or rapid cycle of rapid alternation of depression and mania $[16,17]$. But there is no medical term of bipolar disorder or affective psychosis in traditional Chinese Medicine. Although there is no medical term of bipolar disorder or affective psychosis in traditional Chinese medicine, there are many detailed descriptions about the explanation or discussion of the disease, which are "TCM manic disease" and "TCM depressive disease". Among them, the "crazy disease" is referring to now mania or manic episode of bipolar disorder. The early descriptions of "starts from craziness, sleeps less but does not starve, has high moral integrity, distinguishes wisdom from itself, has high self-esteem, and endures day and night" is very similar to the emotional upsurge, irritability, exaggeration and little sleep of now mania. Therefore, it is considered that the disease is related to internal injury of seven emotions, irregular diet, and congenital heredity. The Seven emotions and six depression, damage to liver qi, induced stagnation of liver qi. The stagnation of Qi continue to transform fire, then following reversal of liver and gall Qi, and opposing of wood and fire, suffering of body fluid, producing phlegm and fire at last. The disturbance of phlegm and turbidity blind the brain orifices and become insanity and madness $[18,19]$. It can be seen that the role of phlegm and fire is very important (19). Previously, liver stagnation was involved. Our conclusion shows that, phlegm fire disturbance to the mind accounted for $48.95 \%$, phlegm and heat stagnation accounted for $20.50 \%$, liver and gall depression heat accounted for $12.37 \%$, the first three accounted for $81.73 \%$, which also showed the pathogenesis of crazy in mania. Not only sthenia syndrome, heat syndrome, but also phlegm fire and phlegm heat play an important role in further development.

Mania or crazy is closely related to fire. Among them, there is a saying that "the more manic all belong to fire" in $<<$ Neijing zhizhenyaodalun $>>$. Therefore, mania belongs to Yang, and it turns to sthenia syndrome. However, if the mania lasts for a long time, it will lead to Qi and Yin injury and deficiency of fire, or qi stagnation and blood stasis. Since then, the two syndromes have also been seen in mania, mostly in the long-term mania. From this description, it can be found that the description of the development and outcome of mania in traditional Chinese medicine is dynamic, reflecting that mania, as an important part of bipolar disorder, can not only alternate or transform with depression, but also develop in other directions, so that the disease is more complex and can also develop into refractory cases [20].

The shortcomings of this study are as follows: (1) this study is a secondary literature study, with certain limitations. (2) The syndrome types in this study have been combined. In fact, although some of them are similar, there are still some differences, which may be subjective; (3) this study only included cases of integrated traditional Chinese and Western medicine, not included cases of non integrated traditional Chinese and Western medicine, inevitably lost some meaningful information.

\section{Fund Projects}

Zhejiang ptovince science and technology program of traditional Chinese medicine (2020ZB040).

\section{References}

[1] McCormick U, Murray B, McNew B. Diagnosis and treatment of patients with bipolar disorder: A review for advanced practice nurses. J Am Assoc Nurse Pract. 2015; 27 (9): 530-42. doi: 10.1002/2327-6924.12275. Epub 2015 Jul 14. 
[2] Morton E, Hole R, Murray G, Buzwell S, Michalak E Experiences of a Web-Based Quality of Life Self-Monitoring Tool for Individuals With Bipolar Disorder: A Qualitative Exploration. JMIR Ment Health 2019; 6 (12): e16121, doi: $10.2196 / 16121$

[3] Chinese society of traditional Chinese medicine. Guidelines for diagnosis and treatment of mental disorders in traditional Chinese medicine [M], 1st edition, Beijing, China traditional Chinese Medicine Press, 2005: 11-15.

[4] Xiong Hong, comparative observation on the treatment of mania by dialectical classification of Chinese and Western Medicine, Electronic journal of clinical medical literature, 2018, 5 (6): 26-27. doi: 10.3877/j.issn.2095-8242.2018.5 (6): 26-27.

[5] Sun Fengli, Gao Zhihan, Zhu Jianfeng, Jin Rui, Li Bingling, Jin Weidong. Strengthening treatment of mania with traditional Chinese medicine: Meta analysis of domestic control study. Asia Pacific Journal of traditional Chinese medicine, 2018: 1 (1): http://www.sciencepublishinggroup.com/j/apjcp.

[6] Sun Fengli, Jin Rui, Zhu Jianfeng, Jin Weidong. Alternative treatment of mania with combination of Chinese and Western medicine: evidence-based medicine evaluation of clinical control study with combination of two western medicine, traditional Chinese medicine, 2018, 7 (6): 391-395. Doi: 10.12677/j.issn.2018. 76065-8242.2018.56.026.

[7] Li Sicong, Jiang Tao, Tong Yujia, etc. A comparative study of TCM Syndromes of 482 cases of bipolar disorder, Beijing Traditional Chinese medicine, 2019, 38 (1): 12-14.

[8] Zhao Yonghou, Zhao Yuping, Chai Jianbo, Yu Ming. Research on literature evaluation of syndrome differentiation and treatment of "crazy disease" based on evidence-based medicine Hunan Journal of traditional Chinese medicine, 2014, 30 (10): 140-142.

[9] Wang yanheng, practical Chinese medicine psychiatry [M], 1st edition, Beijing, people's Health Press, 2000: 529-533.

[10] Wang Yongyan, Lu Zhaolin. Internal medicine of traditional Chinese medicine [M], 1st edition, Beijing, people's Health Press, 2017: 529-533.
[11] Sun Fengli, Yu Fang, song Xinyu, etc. TCM syndrome types of manic mania: Part I: analysis of single syndrome type, 2019, to be published.

[12] Sun Fengli, Yu Fang, song Xinyu, etc. Analysis of TCM syndrome types of manic mania from clinical research of Integrated Chinese and Western medicine: Part I: analysis of multiple syndrome types, 2019, to be published.

[13] Davenport K, Hardy G, Tai S, Mansell W. Individual experiences of psychological-based interventions for bipolar disorder: A systematic review and thematic synthesis. Psychol Psychother 2019 Dec; 92 (4): 499-522.

[14] Shim IH, Lee J, Kim MD, Jung YE, Min KJ, Kwon YJ, Kim JS, Lee K, Woo YS, Nam B, Seo JS, Lee JG, Jon DI, Sohn I, Park $\mathrm{SY}$, Yoon BH, Bahk WM. The prevalence and diagnostic classification of mixed features in patients with major depressive episodes: A multicenter study based on the DSM-5.Int J Methods Psychiatr Res, 2019; 28 (3): e1773.

[15] Tavormina G. Bipolar disorders and bipolarity: the notion of the "mixity". Psychiatr Danub 2019; 31 (Suppl 3): 434-437.

[16] Erol A, Winham SJ, McElroy SL, Frye MA, Prieto ML, Cuellar-Barboza AB, Fuentes M, Geske J, Mori N, Biernacka JM, Bobo WV. Sex differences in the risk of rapid cycling and other indicators of adverse illness course in patients with bipolar I and II disorder. Bipolar Disord 2015; 17 (6): 670-6.

[17] Wehr TA. Bipolar mood cycles and lunar tidal cycles. Mol Psychiatry 2018; 23 (4): 923-931.

[18] Gao Zhiguo. TCM etiology and treatment of bipolar disorder. TCM research, 2017, 30 (11): 5-7.

[19] Li Sicong, Kong Huijun. Analysis of bipolar affective disorder from the perspective of phlegm and fire. Global traditional Chinese medicine, 2018, 11 (10): 1629-1630.

[20] Rong C, Park C, Rosenblat JD, Subramaniapillai M, Zuckerman H, Fus D, Lee YL, Pan Z, Brietzke E, Mansur RB, Cha DS, Lui LMW, McIntyre RS. Predictors of Response to Ketamine in Treatment Resistant Major Depressive Disorder and Bipolar Disorder.Int J Environ Res Public Health, 2018, 15 (4), 771; https://doi.org/10.3390/ijerph15040. 